\title{
THE
}

\section{Interference Suppression in Congested Undersea Environments}

James McGee

Josko Catipovic

Steven Schoenecker

Peter F. Swaszek

University of Rhode Island, swaszek@uri.edu

Follow this and additional works at: https://digitalcommons.uri.edu/ele_facpubs

The University of Rhode Island Faculty have made this article openly available.

Please let us know how Open Access to this research benefits you.

This is a pre-publication author manuscript of the final, published article.

Terms of Use

This article is made available under the terms and conditions applicable towards Open Access Policy Articles, as set forth in our Terms of Use.

\section{Citation/Publisher Attribution}

McGee, James, Josko Catipovic, Steven Schoenecker, and Peter Swaszek. "Interference Suppression in Congested Undersea Environments." Proceedings of the OCEANSS '15 METS/IEEE, Genova, Italy, May 2015, pp. 1-10.

This Article is brought to you for free and open access by the Department of Electrical, Computer, and Biomedical Engineering at DigitalCommons@URI. It has been accepted for inclusion in Department of Electrical, Computer, and Biomedical Engineering Faculty Publications by an authorized administrator of DigitalCommons@URI. For more information, please contact digitalcommons-group@uri.edu. 


\title{
Interference Suppression in Congested Undersea Environments
}

\author{
James McGee, Josko Catipovic and Steven Schoenecker \\ Naval Undersea Warfare Center \\ Newport, RI 02841 \\ Email: james.a.mcgee@navy.mil \\ Email: josko.catipovic@navy.mil \\ Email: steven.schoenecker@navy.mil
}

\author{
Peter Swaszek \\ Department of Electrical, Computer and Biomedical Engineering \\ University of Rhode Island \\ Kingston, RI 02881 \\ Email: swaszek@ele.uri.edu
}

\begin{abstract}
Many acoustic channels suffer from interference which is neither narrowband nor impulsive. This relatively long duration partial band interference can be particularly detrimental to system performance. In operational networks, many "dropped" messages are lost due to partial band interference which corrupts different portions of the received signal depending on the relative position of the interferers, information source and receivers due to the slow speed of propagation. We survey recent work in interference mitigation as background motivation to develop a spatial diversity receiver for use in underwater networks and compare this multi-receiver interference mitigation strategy with a recently developed single receiver interference mitigation algorithm using experimental data collected from the underwater acoustic network at the Atlantic Underwater Test and Evaluation Center. The results indicate that both mitigation strategies are effective: parameterized interference cancellation is most effective at moderate SIRs whereas spatial diversity reconstruction is effective and realizes the most gain at high SIRs. We also apply the parametized interference cancellation to the problem of estimating mutually interfering waveforms when it is desired to know both time domain signals and find that it effectively extracts both mutually interfering linear frequency modulated (LFM) and orthogonal frequency division multiplexing (OFDM) waveforms.
\end{abstract}

\section{INTRODUCTION}

The U. S. Navy operates an undersea cellular network in the Tongue of the Ocean [1]. Known as the Atlantic Undersea Test and Evaluation Center (AUTEC), it consists of 96 acoustic sensors placed over a 60 by 30 kilometer square area and is shown in Fig. 1. As currently configured, approximately 97 percent of transmitted messages are successfully decoded; of the remaining three percent, many are corrupted by acoustic interference arising from active acoustic emissions. For example, Fig. 2 shows the impact of interference on a received data packet. In pane (a), the data packet was received without interference and successfully decoded in contrast to pane (b) where interfering signals are clearly evident and the message was lost. Years of extensive observations of activities in the vicinity of the network demonstrate that the widely separated hydrophones suffer from partial-band interference emanating from multiple spatially separated sources. The nature of this interference is different from the impulsive or narrowband interference typically encountered in other applications. Furthermore, unlike RF communications and acoustic array processing applications where interference is highly correlated in time among the various receivers, in the acoustic network, interference affects different portions of the received signals due to the wide separation of the receivers and the low speed of propagation. The degradation in the received signal is highly variable, depending on the relative position of the interfering signals, information source and receivers as well as the channel conditions. Successful steps to mitigate interference using a single receiver have been reported in [2], and the potential of using multiple receivers to mitigate interference was demonstrated in [3]. The motivation behind this work is to compare the potential benefits that each mitigation strategy provides. We also examine the possibility of estimating both mutually interfering waveforms for the case of receiving LFM and OFDM waveforms simultaneously.

Interference mitigation has a long history in RF communications but the interference is typically impulsive or narrowband [4] and the algorithms are typically developed for a single receiver. Partial band interference is not addressed [2]. The interference mitigation techniques typically exploit the short time or limited frequency span of the interfering signal. Examples of impulsive noise suppression techniques for multi-carrier modulation may be found in [5]-[14] while [15]-[24] address narrowband interference mitigation. Early approaches tended to separate channel estimation and interference detection, and more recent work has focused on jointly estimating the channel and mitigating interference.

Optimum reception is possible given the probability density function of the impulsive noise, but typically the impulsive noise statistics are not available to the receiver [6]. Without a priori knowledge of the impulsive noise statistics, the traditional approach to dealing with impulsive noise has been to mark those symbols hit by impulses as erasures rather than correcting them 


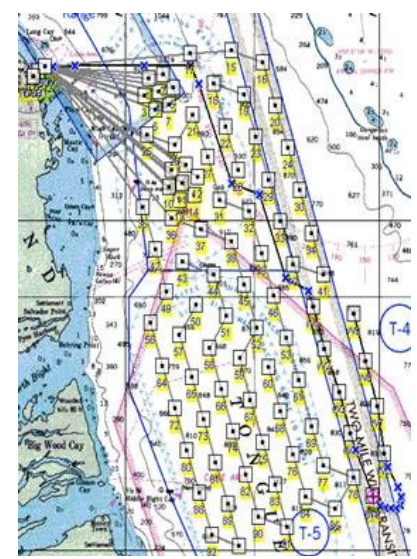

Figure 1: The AUTEC acoustic network.

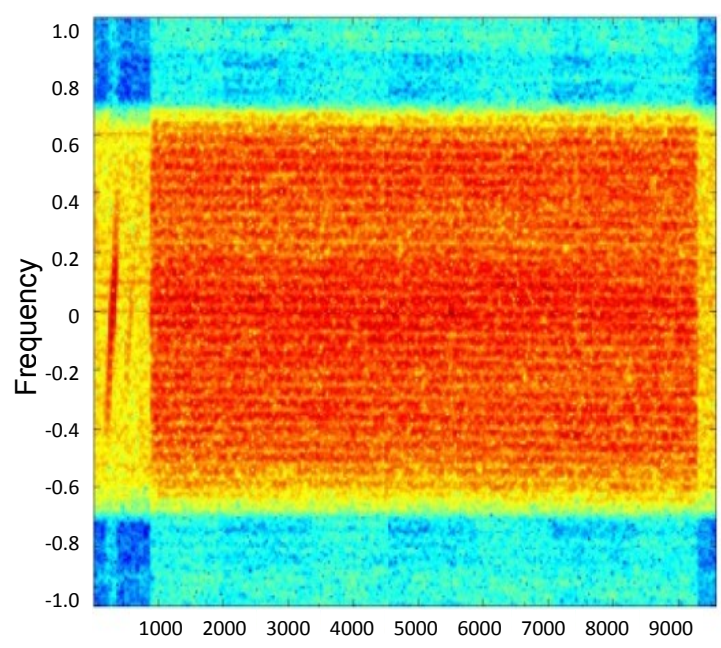

$3000-40005000 \quad 6000700080009000$

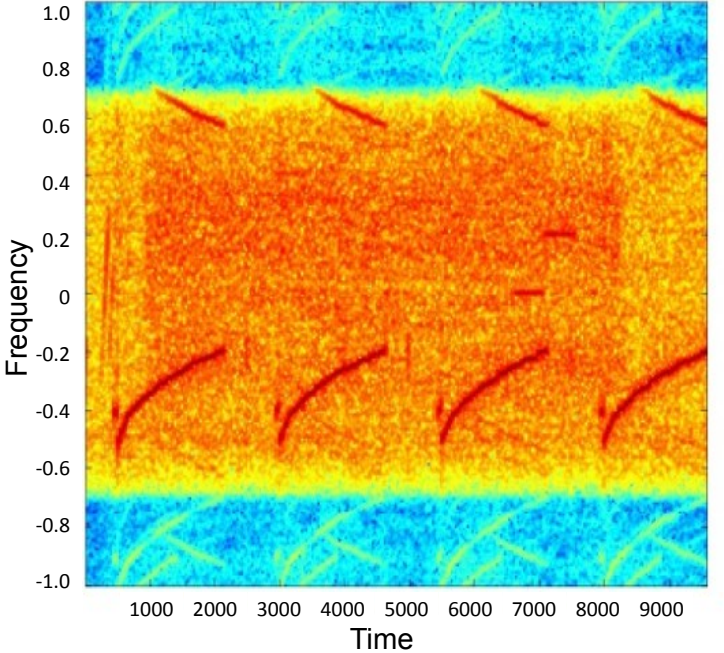

(a)

(b)

Figure 2: Clean packet reception (a) and packet corrupted by acoustic interference (b).

directly since more erasures than errors can be corrected by the same code [6]. The marking of erasures and error-and-erasure decoding have traditionally been performed separately in cascade [6]. The work in [5] applied the traditional cascade approach but explored using error correction coding (soft error protection) and adaptively adjusting the erasures marking threshold (hard error protection) to combat short time duration, high magnitude interference. Supplying channel state information to the decoder enhanced the performance of the applied forward error correction (FEC) code. The erasures marking threshold was adjusted on each of the multicarrier subchannels to protect those subchannels most susceptible to interference. The authors found that combining both the soft and hard error protection was most effective. A low computational complexity algorithm to cancel the impact of impulse noise in the time domain was proposed in [10]. It employed threshold blanking to cancel the impact of impulse noise on the traditional time domain least squares OFDM channel estimate. In [7], a frequency domain strategy to compensate for impulsive noise after OFDM demodulation and channel equalization was proposed as an alternative to the more conventional approach of suppressing the impulsive noise in the time domain. A support vector machine (SVM) algorithm for coherent robust demodulation of OFDM in the presence of impulse noise interfering with the pilot tones was developed in [9]. Compressed sensing techniques were applied to the impulse noise cancellation problem in [11]. Recently, [14] explored passband and baseband clipping approaches for suppressing impulsive noise in Direct Sequence Spread Spectrum Systems in underwater communications.

A narrowband interference canceller was developed in [15] by using a model of the power spectral density of the narrowband interferer as prior information. The canceller estimates the linear minimum mean-square error of the spectral leakage by measuring the narrowband interference on a few active or null OFDM subcarriers. [16] and [17] propose mitigating narrowband interference in OFDM through successive cancellation by applying hard or soft decisions based on the known or estimated second order statistics of the received signal. Using the structure provided by FEC coding, each subcarrier is used to predict the narrowband 
interference contribution on the considered subcarrier in order to subtract out the contribution in an iterative fashion. The most reliable subcarriers are decoded first.

Joint approaches to equalization and interference mitigation may work iteratively such as in [8] or by expanding the states of the decoding algorithm as in [19] and [21]. In [8] applying a clipping and nulling technique at the front of an iterative algorithm significantly reduced the symbol error rate (SER). The iterative decoder further improved performance through a novel syndrome decoding technique which segregated the decoding based on the noise level of the received components. The joint approach to erasure marking and decoding (joint erasure marking Viterbi algorithm (JEVA)) proposed in [6] was motivated by the observation that separation of erasure marking and channel decoding leads to less accurate detection of the impulse noise corrupted symbols because the erasure marker does not take the code structure into account. For a given number of erasures, the JEVA automatically finds the most likely erasure pattern based on the most likely trellis path under the erasure constraint. The algorithm effectively mitigates impulsive noise at the expense of higher complexity. In [21], a simple pilot aided interference detector removes in-band interference. Residual interference that cannot be detected by the pilot aided interference detector is removed by a joint interference detection and decoding scheme. By exploiting the code structure in interference detection, the joint scheme detects most of the symbols suffering residual interference without requiring knowledge of the interference distribution.

A message-passing approach to jointly estimate the channel and mitigate strong co-channel interference of similar form as the desired signal was proposed in [25]. The algorithm, based on belief propagation (BP), which performs statistical inference on graphical models by propagating locally computed beliefs, effectively exploited the non-Gaussian statistics of the interference enabling its detection and suppression without requiring specific knowledge of the interfering signal. Two blind algorithms to mitigate multiple interferers were proposed in [26]: one for the case of strong signal and one for the case of strong interference. Both algorithms find the coefficients of the linear Minimum Mean Square Error filter based on the autocorrelation matrix function of the channel outputs.

Generally, joint approaches provide better performance at the cost of additional computational complexity. Limitations on system performance may be found in [27] for OFDM systems subject to impulsive noise and for multicarrier and single carrier quadrature amplitude modulation (QAM) systems in [28]. The capability of Low Density Parity Check (LDPC) and turbo coding to mitigate burst errors is discussed in [29].

In mobile radio, optimal receiver combining (ORC) [30] is a multi-receiver technique that has been used for years to combat interference. Its performance bounds were developed in [31], but the channel is much less complicated than in underwater communications: it contains no multipath and Doppler effects may be safely ignored. The ORC algorithm assumed the channel was known and the combining was performed on the time domain received waveform. Its original application was to Phase-Shift Keying (PSK) modulation.

Some more recent work has addressed diversity combining in the underwater environment. A receiver that addressed the challenges of the underwater channel and performed joint diversity combining of multiple channel receptions with channel equalization was proposed in [32]. More recently, [33] proposed a multiple-input multiple output system which used space-time coding and iterative decoding techniques to obtain high data rates over shallow-water, medium range channels in the absence of interference. In the 200-300 Hz frequency range, [34] exploited beam diversity among sensors separated by hundreds of meters to improve communication performance.

Whereas processing time and computational complexity are critical drivers in many RF applications (such as mobile phones, digital audio and video broadcasting), underwater communication applications exist (such as submarine to shore message trafficking) where decoding delays are tolerable and vast amounts of computational power are available. Thus, the additional available decoding time and computing power may be employed to untangle the more complicated channel and Doppler effects.

Observations from past field experiments indicate that significant improvement in the reliability of message reception can be realized by mitigating interference. Discussion of the interference typical in the underwater environment is available in [35] and [36].

The work in [2] developed a single receiver parameterized interference cancellation (PIC) algorithm to mitigate partial band interference through a representation of the interference based on a rough estimate of its time duration and bandwidth. The PIC algorithm explicitly canceled the interference without information loss or performance degradation caused by approximations. In [3] we developed a multi-receiver algorithm that relied on the spatial diversity inherent in an acoustic network to facilitate the reconstruction of the transmitted waveform from the clean portions of the received signals. Our algorithm also relied on the known time duration and bandwidth of the interference to perform mitigation. While both approaches rely on prior knowledge of the interference band and time duration, the techniques differ in the use of the knowledge. In [2] prior knowledge aids in the estimation of the interference parameters and facilitates coherent cancellation where as in [3] prior knowledge facilitates the detection and excision of portions of the received signal that suffer from interference. For both approaches, knowledge of the interference parameters does not need to be particularly precise and can be determined in situ. The experiment reported here used Linear Frequency Modulated (LFM) signals as interference. However, both approaches are applicable to any time and band limited signal such as Hyperbolic Frequency Modulated (HFM) waveforms or marine mammal vocalizations. 


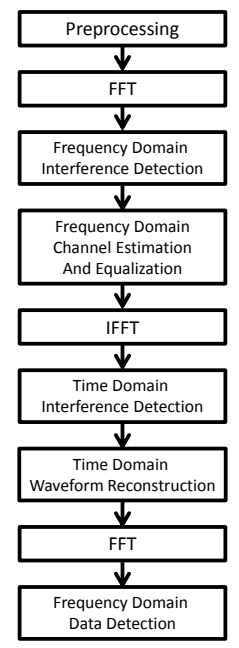

Figure 3: OFDM waveform reconstruction process.

\section{A. Parameterized Interference Cancellation}

The work in [2] developed an iterative single receiver algorithm to mitigate partial band interference of known time duration, $T_{I}$, and interference bandwidth, $B_{I}$. Since the interference is time and band limited, it can be represented by a Fourier-series. The algorithm employs an iterative approach to estimate the $N_{I}=\left\lceil B_{I} T_{I}\right\rceil$ complex coefficients as well as the time delay (with respect to the start of the OFDM block) of the interference. On each iteration, the estimates of the interference and detected symbols from the previous iteration are used to construct a Generalized Likelihood Ratio Test (GLRT) to see if interference is still present. If residual interference is detected, its model parameters are calculated with a maximum-likelihood approach. The modeled interference is than subtracted out, and the channel is equalized and symbol estimation is again attempted. This is repeated until the parity checks are satisfied or some maximum preset iteration limit is reached.

\section{B. Spatial Diversity Reciever}

The spatial diversity receiver identifies portions of the signal suffering from interference on different receivers, removes these portions of the signal and then optimally combines the remaining clean portions of the signal. The process, illustrated Fig. 3, involves switching between the time and frequency domain because interference excision and waveform recombining must be done on equalized time-domain waveforms and all of the information for channel estimation is present in the frequency (or OFDM symbol) domain. Pre-processing synchronizes the received signals and adjusts for Doppler induced dilation or contraction. The signals are then transferred to the frequency domain for removal of any residual Doppler effect, channel equalization and testing for the presence of interference. The frequency domain interference detector compares the signal power on the null subcarriers inside and outside of the interference band to determine if interference is present. Signals having interference are transferred back to the time domain where knowledge of the interference time duration aids in determining which portion of the time domain waveform suffers from interference. Reconstruction of time domain waveform excises time orthogonal interference and averages across portions of the waveform suffering from only noise. If the interference is not time orthogonal (e.g. it occupies the same time bins on all receivers), averaging is performed. The synthesized signal is then transferred back to the frequency domain for data detection.

\section{Comparison of Interference Mitigation Approaches}

The SDR algorithm and the PIC algorithm address the problem of interference in fundamentally different ways. SDR requires multiple receivers while PIC operates on a single receiver and could be extended to multiple receivers. However, it is unclear that the performance gain from adding additional receivers would be significantly better than processing the channel with the highest signal-to-interference ratio (SIR) alone. The SDR approach is to blank out portions of the time domain waveform suffering from interference, while the PIC approach estimates the interference and coherently subtracts it from the desired signal. The SDR approach thus takes advantage of the time orthogonality of the interference whereas PIC does not. In cases where the interference is not time orthogonal, SDR improves performance by appropriately weighting the reconstructed signal based on both the SNR and SIR. Both algorithms use $a$ priori knowledge to aid in frequency domain interference detection. SDR uses $a$ priori knowledge to set the size of the time domain blanking window while in PIC a priori knowledge determines the number of coefficients used to estimate the interference. In SDR, imprecise a priori knowledge leads to some performance degradation because improperly sizing the blanking window results in interference contaminating the signal if the window is too small, or loss in averaging gain if the window is too large. In contrast, for PIC, if the number of coefficients to estimate is too large, 


\begin{tabular}{|l|l|l|}
\hline \multicolumn{2}{|c|}{ OFDM Parameter } & Value \\
\hline Center frequency & $f_{c}$ & $11 \mathrm{kHz}$ \\
\hline Bandwidth & $B$ & $6 \mathrm{KHz}$ \\
\hline \# of subcarriers & $K$ & 1024 \\
\hline \# data subcarriers & $\left|\mathcal{S}_{D}\right|$ & 86 \\
\hline \# pilot subcarriers & $\left|\mathcal{S}_{P}\right|$ & 256 \\
\hline \# null subcarriers & $\left|\mathcal{S}_{N}\right|$ & 96 \\
\hline Symbol Duration & $T$ & $104.68 \mathrm{~ms}$ \\
\hline Symbol Constellation & & BPSK \\
\hline Subcarrier spacing & $\Delta f=1 / \mathrm{T}$ & $9.54 \mathrm{~Hz}$ \\
\hline Guard interval & $T_{g}$ & $24.6 \mathrm{~ms}$ \\
\hline Number of Guard Samples & $N_{g}$ & 240 \\
\hline Synthesized Interference Parameters & Value \\
\hline Center Frequency & $f_{c, I}$ & $11 \mathrm{kHz}$ \\
\hline Bandwidth & $B_{I}$ & $B / 2$ \\
\hline Duration & $T_{I}$ & $T / 3$ \\
\hline
\end{tabular}

Table I: Experiment parameters.

the excess coefficients get little weight. Consequently, there is less loss due to imprecise prior knowledge. Furthermore for PIC, the important parameter in determining the number of coefficients to estimate is the time bandwidth product. Interfering signals with different time durations and bandwidths but a time bandwidth product less than the selected value $N_{I}$ will still be estimated accurately and subtracted. In contrast, SDR requires the time duration and bandwidth to be known separately and its performance is best when applied to signals with the specified time duration and bandwidth.

While SDR requires more detailed prior knowledge than PIC, its performance is less dependent on the SIR as will be seen in the results presented in the next section. PIC relies on accurately estimating the channel and transmitted symbols to aid in estimating and canceling the interference. As the interference gets stronger, channel estimation and symbol detection degrade resulting in poorer estimates of the interference. At loud enough interference levels, the algorithm fails. Because SDR blanks the interference rather than attempting to cancel it, the algorithm is less susceptible to interference induced equalization errors. In contrast to PIC, there is no low SIR threshold where SDR abruptly fails. Its performance is more strongly a function of the background noise and the degree of time orthogonality of the interference than the level of the interference.

\section{EXPERIMENTAL RESUlts}

During May 2014, experiments were conducted at AUTEC to test the interference mitigation algorithms. A multichannel projector was configured for multichannel simultaneous transmission of both interfering and desired acoustic waveforms. The desired OFDM signal consisted of a channel probe, four data packets and a final channel probe while LFM waveforms of various time durations and bandwidths provided the interference. Multiple interfering and desired waveforms were simultaneously transmitted from AUTEC's acoustic transmitters and recorded on more than forty distributed ocean-bottom hydrophones. Examples of the received data packets are shown in Fig 4. The interfering LFM waveform is clearly evident in both spectrograms. In addition to the LFM interference, the third data block suffers interblock interference (IBI) as portions of the surface bounce arrival of the first data2 block coincides with the direct path arrival of the third data block.

Independently processing the third block from the channels shown in Fig 4 without interference suppression resulted in thirteen errors on channel 68 and eight errors on channel 76 where as applying the parameterized interference mitigation algorithm resulted in no errors. Processing the block using maximum ratio combining (MRC) resulted in three errors while applying spatial diversity reconstruction (SDR) resulted in no errors. The distance between these receivers is approximately 4,000 yards.

In order to test the performance of the algorithms at different interference and noise level, a data set of 1,500 synthesized received signals was created from the interference and noise sampled during the experiment. The pseudo-experimental interference was created by windowing the appropriate portions of the received direct path LFM signals and amplifying them to the appropriate interference level.. Similarly, noise from portions of the experiment when no signal or interference was present was amplified to the appropriate level to vary the signal to noise ratio. The amplified interference and noise was added to received OFDM blocks in which the symbols were all detected correctly prior to decoding. For testing of the SDR and MRC algorithms, the interference was time orthogonal on the two receivers. Table ?? provides details about the experiment.

The results of the processing the pseudo-experimental signals prior to decoding are shown in 5 for signal channel processing, MRC and SDR. A clairvoyant (shown in cyan diamonds) and non-clairvoyant (shown in blue circles) SDR algorithm were tested. The clairvoyant algorithm always excised the correct portion of the signal suffering interference whereas the non-clairvoyant algorithm had to detect the presence of interference as well as select the portion of the received signal to excise. At low SIRs (when the interference is large), there is a clear benefit to excising the interference for all values of SNR but more dramatic at higher SNRs. As the interference power approaches the noise power, the benefit of excising the interference diminishes and the loss from not averaging over the noise becomes apparent. This is demonstrated at higher SIRs in the results for the clairvoyant SDR receiver where applying the algorithm, even with perfect knowledge of the time window suffering interference, results in degraded performance as compared to MRC . However, the interference detection threshold can be set to smoothly transition 

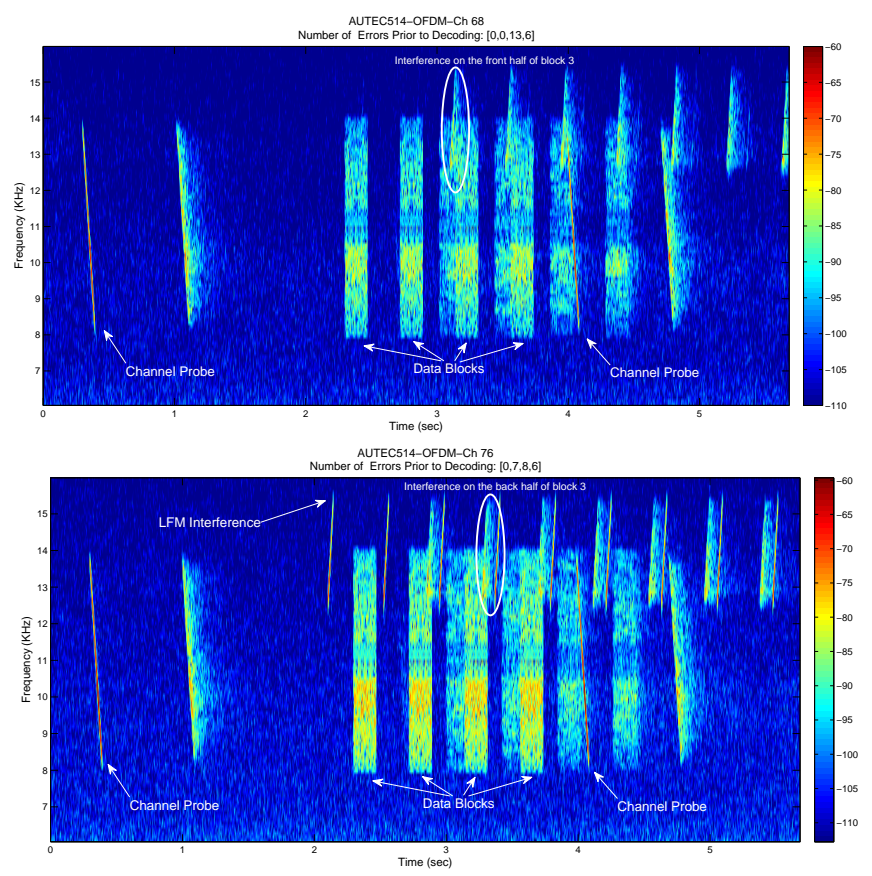

(a)

(b)

Figure 4: The OFDM signals received on channels 68 (panel a) and 76 (panel b) during an experiment at AUTEC in May 2014. Note that LFM interference corrupts for the beginning of block 3 of the data packet on channel 68 and the end of the same block on channel 76 . The receivers are separated by approximately 4,000 yards.

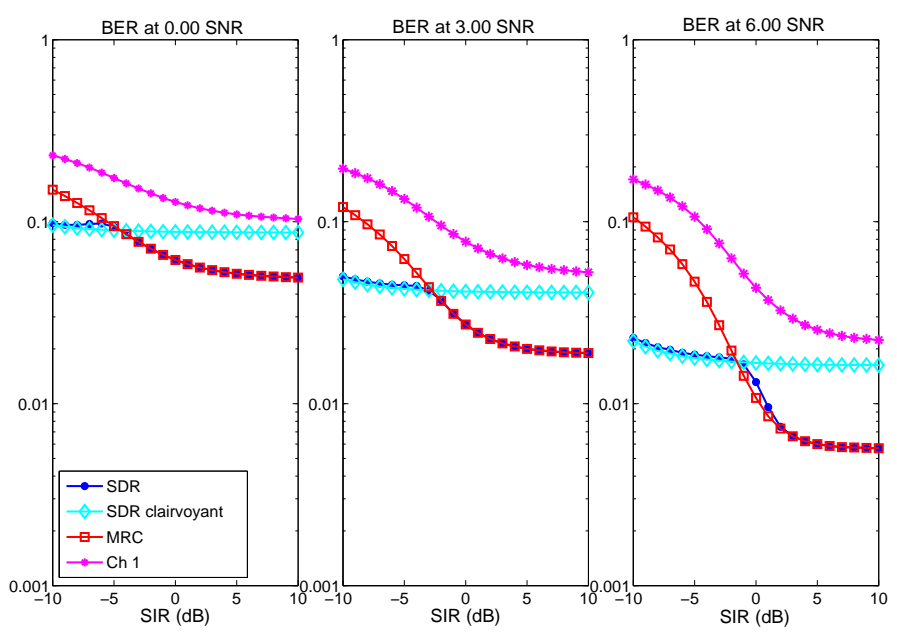

Figure 5: Bit error rate performance as a function of SIR for the different mitigation strategies.

between the SDR and MRC combining strategies. By only declaring interference when it is sufficiently large, the SDR receiver without clairvoyance exhibits the same performance as MRC at high SIRs.

Fig. 6 shows the bit error rate after decoding for the various receivers. (The single channel uncoded bit error rate is also shown for comparison). The single channel PIC algorithm (green triangles) is effective at moderate SIR levels. This is the region of where Fig. 5 indicated the most effective strategy was to transition SDR to MRC. The PIC technique is ineffective at high SIRs; precisely the region where the SDR gains are most dramatic. Thus, the two approaches are complimentary and an effective multi-channel receiver strategy would be to adaptively utilize both techniques.

\section{A. Extraction of an LFM Signal from an OFDM Signal}

From a communications perspective, the LFM signal corrupting a block of OFDM data is undesirable and the information gained about it in the interference estimation process is discarded. However, other applications exist where two partially known 


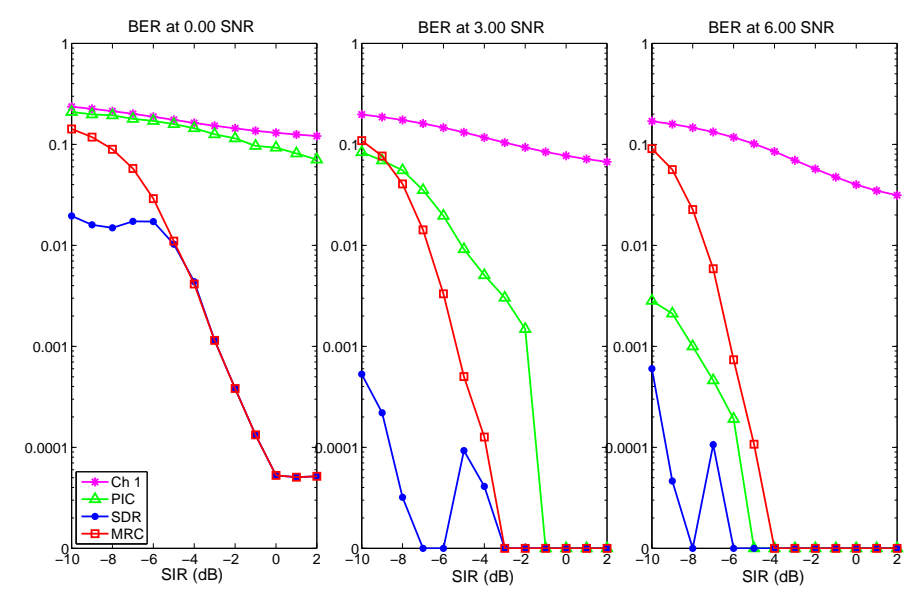

Figure 6: Coded bit error rate performance as a function of SIR for the different mitigation strategies.

signals interfere with one another and it is desirable to obtain accurate time domain estimates of both received signals. The PIC algorithm provides one possible method to achieve this. As an initial approach to looking at this problem, we examined mutually interfering LFM and OFDM signals. We transferred the PIC algorithm frequency domain estimate of the LFM interference to the time domain and compared it to the known received interfering waveform. Figures 7 - 9 compare the estimated LFM interference to the actual received LFM interfering waveform as well as the original transmitted LFM signal. The results are typical for the mutual LFM and OFDM data we observed. The top panel of Figure 7 shows the spectrogram and times series of a received OFDM signal block with an interfering LFM signal that is $6 \mathrm{~dB}$ lower than the OFDM signal power. The bottom panel shows the spectrogram and time series of the estimated LFM signal. Comparison of the actual and estimated time series of the received signal is shown in Figure 8. Clear gain is seen in the matched filter output of the extracted LFM signal over the original received signal (LFM + OFDM) as shown in Figure 9. The estimate of the OFDM waveform is not shown but its accuracy is assured because it was decoded without error. The results indicate that using the PIC algorithm to estimate mutually interfering waveforms is possible and beneficial.

\section{SUMMARY}

Many acoustic channels suffer from interference which is neither narrowband nor impulsive. This relatively long duration partial band interference can be particularly detrimental to system performance. However, some parameters of the interference are usually known or can be acquired and exploited to facilitate interference cancellation on a single receiver. For multiple receivers, the prior knowledge can be used to exploit the detection and excision of portions of the received signals suffering from interference so that they can be combined more effectively. While the single receiver technique relies on accurately estimating and subtracting the interference, the multi-receiver technique exploits the slow speed of sound propagation in water and the geographical extent of networks, to combine clean portions of the received signals. The experimental results from AUTEC demonstrate that both techniques are effective mitigation strategies. Parameterized interference cancellation is most effective at moderate SIRs whereas spatial diversity reconstruction is effective and realizes the most gain at high SIRs. The two approaches are complimentary and an effective multi-channel receiver strategy would be to adaptively utilize both techniques. Applying the parametized interference cancellation to the problem of estimating two mutually interfering waveforms is also possible as demonstrated by the effective estimation of both mutually entangled LFM and OFDM waveforms.

\section{REFERENCES}

[1] R. Hayford, D. Nagle, and J. Catipovic, "Undersea cellular network, Tongue of the Ocean Bahamas," in Proc. of the 4th ACM Workshop on Underwater Network (WuWNet), Berkerly, 2009.

[2] Z. Wang, S. Zhou, J. Catipovic, and P. Willett, "Parameterized cancellation of partial-band partial-block-duration interference for underwater acoustic OFDM," IEEE Transactions on Signal Processing, vol. 60, no. 4, pp. 1782-1795, 2012.

[3] J. McGee, J. Catipovic, and P. Swazsek, "Leveraging spatial diversity to mitigate partial band interference in undersea networks through waveform reconstruction," in to appear in Proc. of MTS/IEEE OCEANS Conf., 2014.

[4] J. Laster and J. Reed, "Interference rejection in digital wireless communications," IEEE Signal Process. Mag., vol. 14, no. 3, pp. $37-62$, 1997.

[5] T. Zogakis, P. Chow, J. Aslanis, and J. Cioffi, "Impulse noise mitigation strategies for multicarrier modulation," in Proc. Int. Conf. Commun., 1993, pp. 784-788.

[6] T. Li, W. H. Mow, and M. H. Siu, "A joint approach to erasure marking and Viterbi decoding for impulsive noise channels," in Proc. IEEE Workshop on Signal Process, 2003.

[7] S. Zhidkov, "Impulsive noise suppression in OFDM-based communication systems," IEEE Trans. Consum. Electron., vol. 49, no. 4, pp. 944-948, 2003.

[8] A. Mengi and A. Vinck, "Successive impulsive noise suppression in OFDM," in Proc. IEEE Int. Symp. on Power Line Commun. Appl., 2010. 

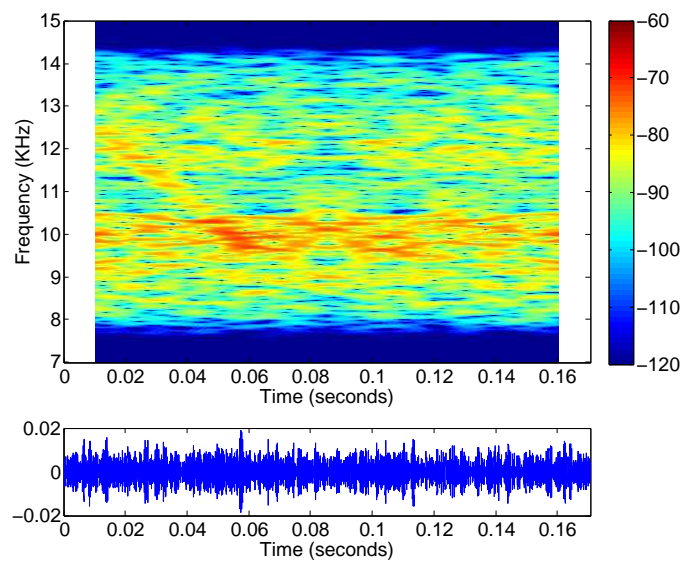

(a)
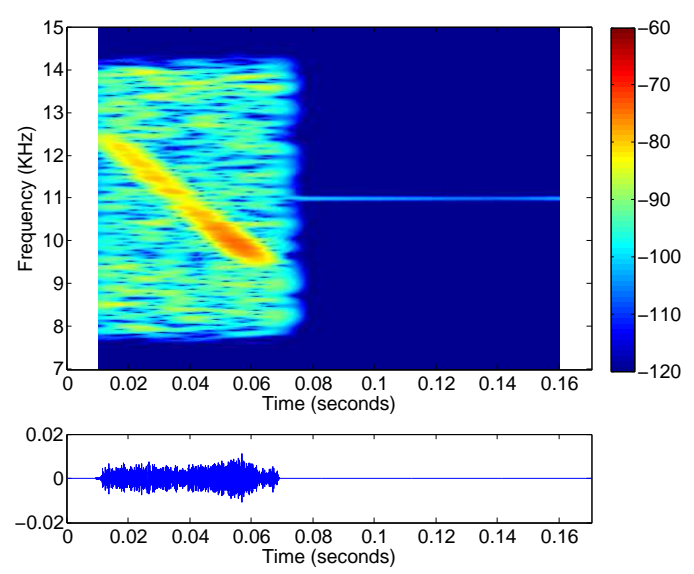

(b)

Figure 7: Extraction of an LFM signal from an OFDM block.

[9] M. F.-G. García, J. Rojo-Álvarez, F. Alonso-Atienza, and M. Martínez-Ramón, "Support vector machines for robust channel estimation in OFDM," IEEE Signal Process. Lett., vol. 13, no. 7, pp. 397-400, 2006.

[10] X. Yu, P. Lin, Z. He, and W. Wu, "OFDM channel estimation with impulse noise cancellation," in Proc. Int. Conf. on Wireless Commun., 2007, pp. 330-333.

[11] G. Caire, T. Al-Naffouri, and A. Narayanan, "Impulse noise cancellation in OFDM: An application of compressed sensing," in Proc. IEEE Int. Symp. Inf. Theory, 2008, pp. 1293-1297.

[12] K. Pelekanakis, H. Liu, and M. Chitre, "An algorithm for sparse underwater acoustic channel identification under symmetric alpha-stable noise," in Proc. of MTS/IEEE OCEANS Conf., 2011.

[13] J. Armstrong and H. A. Suraweera, "Decision directed impulse noise mitigation for OFDM in frequency selective fading channels," in Proc. GLOBALCOM, 2004, pp. 3536-3540.

[14] X. Xu, S. Zhou, H. Sun, A. K. Morozov, and Y. Zhang, "Impulsive noise suppression in per survivor processing based DSSS systems," in Proc. IEEE Oceans Conference, St John's Newfoundland Canada, September 2014.

[15] R. Nilsson, F. Sjoberg, and J. LeBlanc, "A rank-reduced LMMSE canceller for narrowband interference suppression in OFDM-based system,” IEEE Trans. Commun., vol. 51, no. 12, pp. 2126-2140, 2003.

[16] D. Darsena, "Successive narrowband interference cancellation for OFDM systems," IEEE Commun. Lett., vol. 11, pp. 73-75, 2007.

[17] D. Darsena and F. Verde, "Successive NBI cancellation using soft decisions for OFDM systems," IEEE Signal Process. Lett., vol. 15, pp. 873-876, 2008.

[18] A. Jeremic, T. A. Thomas, and A. Nehorai, "OFDM channel estimation in the presence of interference," IEEE Trans. Signal Process., vol. 52, no. 12, pp. 3429-3439, 2004.

[19] M. Morelli and M. Moretti, "Channel estimation in OFDM systems with unknown interference," IEEE Trans. Wireless Commun., vol. 8, no. 10, pp. $5338-5347,2009$.

[20] _ " "Improved decoding of BICM-OFDM transmissions plagued by narrowband interference," IEEE Trans. Wireless Commun., vol. 10, no. 1, pp. 20-26, 2011.

[21] T. Li, W. H. Mow, V. Lau, M. Siu, R. Cheng, and R. Murch, "Robust joint interference detection and decoding for OFDM-based cognitive radio systems with unknown interference," IEEE J. Sel. Areas Commun., vol. 25, no. 3, pp. 566-575, 2007.

[22] A. Gomaa, K. M. Z. Islam, and N. Al-Dhahir, "Two novel compressive-sensing algorithms for NBI detection in OFDM systems," in Proc. Int. Conf. on Acoust., 2010, pp. 3294-3297. 

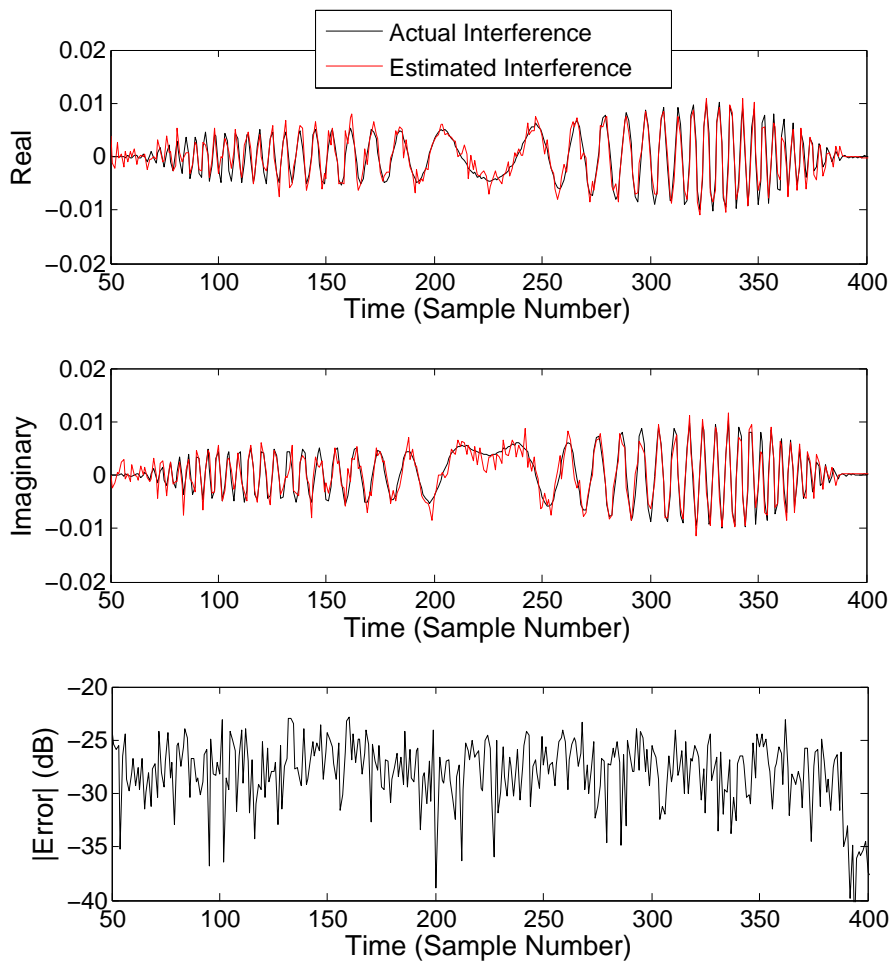

Figure 8: Actual received and estimated LFM signal.

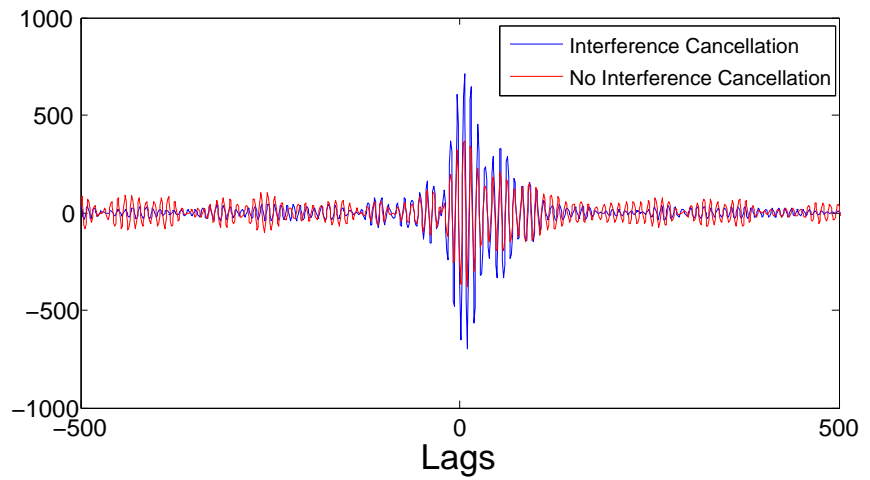

Figure 9: Matched filter output with and without interference cancellation.

[23] T. Al-Naffouri, A. Quadeer, and G. Caire, "Impulsive noise estimation and cancellation in DSL using orthogonal clustering," in Proc. IEEE Int. Symp. Inf. Theory, 2011, pp. 2841-2845.

[24] K. Fazel, "Narrow-band interference rejection in orthogonal multicarrier spread-spectrum communications," in Third Annual International Conference on Universal Personal Communications, 1994, pp. 46-50.

[25] Y. Zhu, D. Guo, and M. L. Honig, "A message-passing approach for joint channel estimation, interference mitigation, and decoding," IEEE Trans. Wireless Commun., vol. 8, no. 12, pp. 6008-6018, 2009.

[26] F. He, X. Wang, T. Yang, B. Hu, and R. Liu, "Two blind algorithms of MMSE filter based on second-order statistics in MIMO systems with multiple interferences," in Wireless Communications and Signal Processing (WCSP), 2011.

[27] S. V. Zhidkov, "Performance analysis and optimization of OFDM receiver with blanking nonlinearity in impulsive noise environment," IEEE Trans. Vehicular Technology, vol. 55, no. 1, pp. 234-242, Jan. 2006Jan.

[28] M. Ghosh, "Analysis of the effect of impulse noise on multicarrier and single carrier QAM systems," IEEE Trans. Commun., vol. 44, no. 2, pp. 145-147, 1996.

[29] S. J. Johnson, Iterative Error Correction: Turbo, Low Density Parity-Check and Repeat Accumulate Codes. New South Wales: Cambridge University Press, 2010. 
[30] J. H. Winters, "Optimum combining in digital mobile radio with cochannel interference," no. 2, 1984, pp. 528-539.

[31] M. Chiani, M. Z. Win, A. Zanella, R. K. Mallik, and J. H. Winters, "Bounds and approximations for optimum combining of signals in the presence of multiple cochannel interferers and thermal noise," IEEE Transactions on Communications, vol. 51, no. 2, pp. 296-306, Feb. 2003.

[32] M. Stojanovic, J. A. Catipovic, and J. G. Proakis, "Adaptive multichannel combining and equalization for underwater acoustic communications," J. Acoust. Soc. Amer., vol. 94, no. 3, pp. 1621-1631, 1993.

[33] S. Roy, T. M. Duman, V. McDonald, and J. G. Proakis, "High rate communication for underwater acoustic channels using multiple transmitters and space-time coding: Receiver structures and experimental results," IEEE J. Ocean. Eng., vol. 32, no. 3, pp. 663-688, Jul. 2007.

[34] H.-C. Song, "Long-range acoustic communication in deep water using a towed array: Beam diversity," in OCEANS, Yeosu 2012.

[35] J. Ward, M. Fitzpatrick, N. Dimarzio, D. Moretti, and R. Morrizzey, "New algorithm for open ocean mammal monitoring," in Proc. MTS/IEEE OCEANS Conf., 2000.

[36] J. Catipovic, "Robust acoustic communication with underwater vehicles in noisy and jammed shallow water environments," in OCEANS 1992: Mastering the Oceans Through Technology, 1992. 